\title{
Editorial: Secondary vs. Idiopathic Autism
}

\author{
Manuel F. Casanova ${ }^{1 *}$, Emily L. Casanova ${ }^{1}$, Richard Eugene Frye ${ }^{2}$, Carolina Baeza-Velasco ${ }^{3}$, \\ Janine M. LaSalle ${ }^{4}$, Randi Jensen Hagerman ${ }^{5}$, Stephen W. Scherer ${ }^{6}$ and Marvin R. Natowicz ${ }^{7}$ \\ 1 School of Medicine Greenville, University of South Carolina, Greenville, SC, United States, ${ }^{2}$ Phoenix Children's Hospital, \\ Phoenix, AZ, United States, ${ }^{3}$ Université Paris Descartes, Île-de-France, France, ${ }^{4}$ University of California, Davis, Davis, CA, \\ United States, ${ }^{5}$ Department of Pediatrics, UC Davis School of Medicine, Sacramento, CA, United States, ${ }^{6}$ University of \\ Toronto, Toronto, ON, Canada, ${ }^{7}$ Cleveland Clinic, Cleveland, $\mathrm{OH}$, Canada
}

Keywords: autism spectrum disorder, idiopathic autism, secondary autism, Fragile X syndrome, anxiety, seizures

\section{Editorial on the Research Topic}

Secondary vs. Idiopathic Autism

This Research Topic consists of four articles, contributed by 30 authors, each focusing on various aspects of secondary autism spectrum disorder (ASD). The articles in this Research Topic discuss some of the controversies and progress made in how to assess a child with secondary ASD. They discuss the utility of using genetic and metabolic tests, as well as combinations of the same. They also grapple with the relationship of intellectual disability (ID) and other symptoms in patients with secondary ASD.

The diagnostic boundaries of the behavioral phenotype that define ASD are fairly broad due to the large variability that is observed in symptom types, onset, and severity. This variability serves as an index of etiological heterogeneity for a group of complex conditions where multiple genetic/ environmental hits provide an additive burden to symptom expression. Within this spectrum of disorders our Research Topic examines a subpopulation of patients where the ASD phenotype occurs and a specific cause can be identified (1). These cases are often referred to as "secondary" ASD as a way of distinguishing them from "idiopathic" ASD. While there is some variation of the estimates, about $85 \%$ of individuals with ASD are termed as having idiopathic ASD with about $15 \%$ diagnosed with secondary ASD (2). Cases of secondary ASD display significant etiological diversity and, sometimes, more severe behavioral problems than individuals having idiopathic ASD $(3,4)$. Well documented causes of secondary ASD include conditions such as tuberous sclerosis, down syndrome, Fragile X syndrome (FXS), and some congenital infections (e.g., cytomegalovirus) (5-7).

Differentiating between idiopathic and secondary ASD is essential when drawing conclusions regarding pharmacological and behavioral treatments among published studies (8). Clinical study design benefits from the selection of homogeneous populations, those without prognostically distinct subgroups, as it makes it easier to assess treatment efficacy (vide infra, (9)]. Indeed, a thorough understanding of the different clinical presentations, natural history and even life expectancies of persons with ASD should go beyond the influence of traditional variables and use relevant classifiers of biomedical and sociocultural differences that may have an impact on the health and wellbeing of the studied subject population.

Genomic scanning is beginning to elucidate the underlying genetic architecture that confers ASD susceptibility. Chromosomal and penetrant gene abnormalities are common, contributing to about $5 \%-15 \%$ of ASD cases in some estimates (10). By way of contrast, de novo chromosomal copy number variants, important contributors to lower IQ, and diminished motor skills (11), are seen in 
approximately $5 \%$ of persons diagnosed with idiopathic ASD (Glinton and Elsea). Many additional cases have DNA sequence variants in genes whose pathology and function provide an uncertain contribution to ASD. These genetic modifications are described as variants with unknown significance (VUS) (12).

For disorders associated with secondary ASD, there is a substantial increase in the prevalence rate of ASD as compared to the general population. The underlying reason for this association is complex and may include a disruption of brain function caused by the superimposed pathology, the possible linkage between ASD risk genes and those of the secondary condition (e.g., ID), and, for some, the commonality in the expression of seizures and their effects on brain development (4, Thurm et al.). Establishing a diagnosis of ASD in this patient population is complicated by the fact that traits of ASD often exhibit a phenotypic and genetic overlap with symptoms of other disorders. It is therefore unsurprising that affected individuals often live with challenges that may confound behavioral observations (Thurm et al.). Indeed, these patients may commonly present with ID, seizures and/or anxiety in addition to comorbid ASD symptoms (11). Identification in these cases often depends on clinical suspicion, especially in severe phenotypes, and both metabolic and genome-wide screening (Glinton and Elsea).

We suggest a concerted effort at developing integrated surveys of clinical, metabolic, and genomic data as a background tool for patient stratification. By establishing a proper diagnosis these tools would aid in understanding underlying pathogenetic mechanisms, in customizing medical/treatment decisions, in offering explanations towards some of the patients' behaviors and, possibly, in providing knowledge helpful in guiding parents and professionals regarding needed school and community services. This integrated approach complements, when necessary, the recommended general developmental screening proposed by the American Academy of Pediatrics (12), keeping in mind that early intervention can and does influence outcomes (13).

Potter et al. reported a clinical trial of sertraline, a selective serotonin reuptake inhibitor, in nonsyndromic ASD. A previous controlled trial of low dose sertraline in children 2 to 6 years old with FXS had demonstrated significant benefit of sertraline compared to placebo in developmental measures on the Mullen Scales of Early Development, especially in the $60 \%$ who has ASD in addition to FXS (14). However, Potter et al. performed a randomized, double-blind, placebo-controlled study $(n=32$ sertraline, $\mathrm{n}=26$ controls) in children with ASD without FXS aged 24-72 months that showed no benefit in primary or secondary outcome measures of language development.

In $\mathrm{ASD}$, the heterogeneity observed along a spectrum of clinical presentations and natural histories is a sobering reminder of the difficulty ingrained in making comparisons among different patient populations. It is not surprising that results of studies using populations of syndromic [FXS, (14)] and idiopathic ASD (Potter et al.) may not reproduce each other. In these cases, large and well characterized study populations are needed in order to perform secondary analyses that could help elucidate differences in severity among the studied populations and better capture individual differences within and between subgroups of autistic individuals. This study concurs with the majority of clinical trials on selective serotonin reuptake inhibitors which show very mixed results and overall suggest that these medications may not be appropriate for children with ASD (15).

Glinton and Elsea offer a comprehensive review of metabolomics and the use of targeted/untargeted assays and their possible role in the early screening and diagnosis of secondary ASD caused by inborn errors of metabolism. The review also introduces the reader to the emerging field of nonspecific metabolic disturbances and how they can lead to clues on mechanisms and treatment. Considering the large variety of metabolic disturbances identified in ASD, the authors question the extent to which any biomarker would accurately assess risk or guide individual treatment. The answer to the question may lie in the use of untargeted assays in order to broadly survey the multiple aspects of the metabolism of each individual patient. The use of biomarkers as metabolic measurements to improve ASD diagnosis and identification of underlying causes is a promising research tool in the hands of physicians but requires specialized skills for proper interpretation (16).

Thurm et al. consider the impact of ID on the diagnosis of ASD. The authors provide a review of the conceptual evolution of autism diagnosis and the different tools used for this purpose. They propose the use of operationalized criteria for making the clinical distinction between ID with and without ASD and provide specific recommendation for testing depending on the degree of ID. The authors consider that the presence of ID in ASD is one of the strongest indicators that an associated condition may be present.

Lovato et al. clarifies the relevance of genetic variants of purported unknown significance (VUS). Some of these variants are genes associated with ASD, but the altered region lacks functional data linking it to pathogenicity. Other VUS are located in genes having few associations with ASD, but may seem relevant to the clinical phenotype. This review summarizes a strategy for obtaining information about VUS by focusing on both clinical phenotyping and a genetic curation process. It is hoped that advanced understanding of these genetic variants may help clarify risk and guide individual treatment options.

\section{AUTHOR CONTRIBUTIONS}

All authors listed have made a substantial, direct, and intellectual contribution to the work, and approved it for publication.

\section{ACKNOWLEDGMENTS}

To the always helpful Frontiers team whose organizational skills and understanding made this Research Topic possible. 


\section{REFERENCES}

1. Faras H, Al Ateeqi N, Tidman L. Autism spectrum disorders. Ann Saudi Med (2010) 30(4):295-300. doi: 10.4103/0256-4947.65261

2. National Institute of Health. About autism. National Human Genome Research Institute. Updated May, 2019. https://www.genome.gov/GeneticDisorders/Autism.

3. Tammimies K, Marshall CR, Walker S, Kaur G, Thiruvahindrapuram B, Lionel AC, et al. Molecular diagnostic yield of chromosomal microarray analysis and whole-exome sequencing in children with autism spectrum disorder. JAMA (2015) 314(9):895-903. doi: 10.1001/jama.2015.10078

4. Wiznitzer M. Autism and tuberous sclerosis. J Child Neurol (2004) 19(9):6759. doi: 10.1177/08830738040190090701

5. Moss J, Richards C, Nelson L, Oliver C. Prevalence of autism spectrum disorder symptomatology and related behavioural characteristics in individuals with Down syndrome. Autism (2013) 17(4):390-404. doi: $10.1177 / 1362361312442790$

6. Wheeler AC, Mussey J, Villagomez A, Bishop E, Raspa M, Edwards A, et al. DSM-5 changes and the prevalence of parent-reported autism spectrum symptoms in Fragile X syndrome. J Autism Dev Disord (2015) 45(3):81629. doi: 10.1007/s10803-014-2246-z

7. Rizzo R, Pavone P. Aripiprazole for the treatment of irritability and aggression in children and adolescents affected by autism spectrum isorders. Expert Rev Neurother (2016) 16:867-74. doi: 10.1080/14737175.2016.1211007

8. Devlin B, Scherer SW. Genetic architecture in autism spectrum disorder. Curr Opin Gent Dev (2012) 22:229-37. doi: 10.1016/j.gde.2012.03.002

9. Buja A, Volfovsky N, Krieger AM, Lord C, Lash AE, Wigler M, et al. Damaging de novo mutations diminish motor skills in children on the autism spectrum. Proc Natl Acad Sci (2018) 115(8):E1859-66. doi: 10.1073/ pnas. 1715427115

10. Schaaf CP, Huda Z. Solving the autism puzzle a few pieces at a time. Neuron. (2011) 70:806-8. doi: 10.1016/j.neuron.2011.05.025
11. Niu M, Han Y, Dy ABC, Du J, Jin H, Qin J, et al. Autism symptoms in Fragile X syndrome. J Child Neurol (2017) 32(10):903-9. doi: 10.1177/ 0883073817712875

12. Hyman SL, Levy SE, Myers SM, Council on Children with Disabilities, Section on Developmental and Behavioral Pediatrics. Identification, evaluation, and management of children with autism spectrum disorder. Pediatrics (2019) 145 (1), e20193447. doi: 10.1542/peds.2019-3447

13. Zwaigenbaum L, Bauman ML, Choueiri R, Kasari C, Carter A, Granpeesheh $\mathrm{D}$, et al. Early intervention for children with autism spectrum disorder under 3 years of age: recommendations for practice and research. Pediatrics (2015) 136 (suppl 1):S60-81. doi: 10.1542/peds.2014-3667E

14. Greiss Hess L, Fitzpatrick SE, Nguyen DV, Chen Y, Gaul KN, Schneider A, et al. A randomized, double-blind, placebo-controlled trial of low-dose sertraline in young children with Fragile X syndrome. J Dev Behav Pediatr (2016) 37(8):619-28. doi: 10.1097/DBP.0000000000000334

15. Williams K, Brignell A, Randall M, Silove N, Hazell P. Selective serotonin reuptake inhibitors (SSRIs) for autism spectrum disorders (ASD). Cochrane Database Syst Rev (2013) (8), CD004677. doi: 10.1002/14651858.CD004677.pub3

16. Frye RE, Vassall S, Kaur G, Lewis C, Karim M, Rossignol D. Emerging biomarkersin autism spectrum disorder: a systematic review. Ann Transl Med (2019) 7(23):792. doi: 10.21037/atm.2019.11.53

Conflict of Interest: The authors declare that the research was conducted in the absence of any commercial or financial relationships that could be construed as a potential conflict of interest.

Copyright (C) 2020 Casanova, Casanova, Frye, Baeza-Velasco, LaSalle, Hagerman, Scherer and Natowicz. This is an open-access article distributed under the terms of the Creative Commons Attribution License (CC BY). The use, distribution or reproduction in other forums is permitted, provided the original author(s) and the copyright owner(s) are credited and that the original publication in this journal is cited, in accordance with accepted academic practice. No use, distribution or reproduction is permitted which does not comply with these terms. 